\title{
Jets and high $p_{T}$ hadrons in dense matter: recent results from STAR
}

\author{
Peter Jacobs ${ }^{* \dagger}$ and Jennifer Klay ${ }^{* \dagger}$ \\ ${ }^{*}$ Lawrence Berkeley National Laboratory, 1 Cyclotron Road, Berkeley CA 94720 \\ ${ }^{\dagger}$ for the STAR Collaboration
}

\begin{abstract}
We review recent measurements of high transverse momentum (high $p_{T}$ ) hadron production in nuclear collisions by the STAR Collaboration at RHIC. The previously observed suppression in central $\mathrm{Au}+\mathrm{Au}$ collisions has been extended to much higher $p_{T}$. New measurements from $\mathrm{d}+\mathrm{Au}$ collisions are presented which help disentangle the mechanisms responsible for the suppression. Inclusive single hadron spectra are enhanced in $\mathrm{d}+\mathrm{Au}$ relative to $\mathrm{p}+\mathrm{p}$, while two-particle azimuthal distributions are observed to be similar in $\mathrm{p}+\mathrm{p}, \mathrm{d}+\mathrm{Au}$ and peripheral $\mathrm{Au}+\mathrm{Au}$ collisions. The large suppression of inclusive hadron production and absence of the away-side jet-like correlations in central $\mathrm{Au}+\mathrm{Au}$ collisions are shown to be due to interactions of the jets with the very dense medium produced in these collisions.
\end{abstract}

\section{INTRODUCTION}

High energy partons propagating through matter are predicted to lose energy via induced gluon radiation, with the magnitude of the energy loss depending linearly on the color charge density of the matter[1]. This phenomenon may provide a sensitive probe of the medium generated in high energy heavy ion collisions, where a Quark-Gluon Plasma (QGP) is expected to form if sufficiently high energy density is achieved. The high energy partons in such collisions result from the hard scattering of quarks or gluons from the incoming nuclei and are observed experimentally as correlated "jets" of hadrons having large transverse momentum $\left(p_{T}\right)$ with respect to the beam direction. The experimental challenge is to measure partonic energy loss ("jet quenching") in the extremely complex environment of high energy nuclear collisions.

In these talks we discuss recent progress towards the measurement of jet quenching in high energy nuclear collisions by the STAR experiment at the Relativistic Heavy Ion Collider (RHIC) at Brookhaven National Laboratory. At the values of jet $E_{T}$ accessible at RHIC energies with the currently achieved integrated luminosities, full jet reconstruction with good energy resolution is difficult. We therefore utilize known features of jet fragmentation to study jet quenching, in particular the inclusive spectrum of high $p_{T}$ ("leading") hadrons and the angular correlation of pairs of high $p_{T}$ hadrons. We compare their distributions in centrality-selected $\mathrm{Au}+\mathrm{Au}$ collisions to those in $\mathrm{d}+\mathrm{Au}$ and non-singly diffractive (NSD) $\mathrm{p}+\mathrm{p}$ collisions, all at $\sqrt{s_{\mathrm{NN}}}=200 \mathrm{GeV}$. Unless otherwise specified, the results reported here are for unidentified charged hadrons, measured in the large cylindrical STAR Time Projection Chamber with a 0.5T solenoidal magnetic field[2]. Related results from STAR are discussed in [3, 4]. 

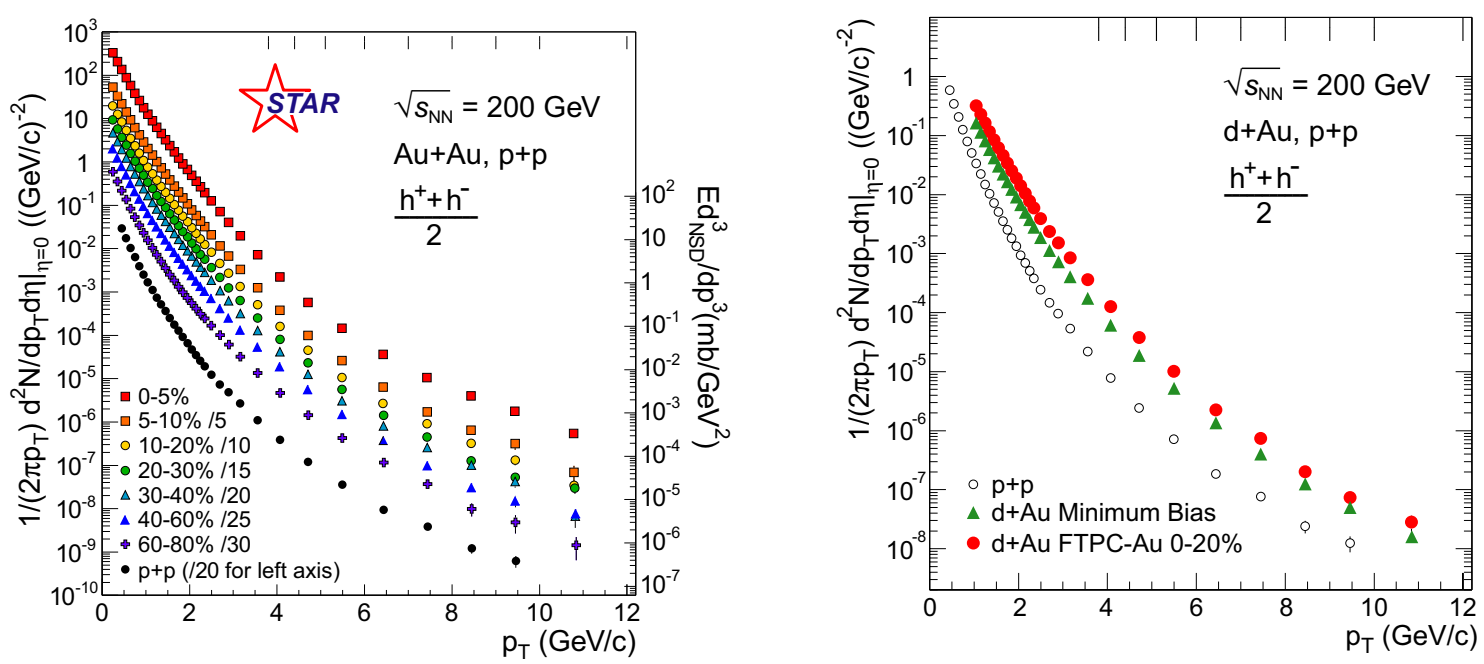

FIGURE 1. Invariant inclusive $p_{T}$ distributions of charged hadrons at $\sqrt{s_{\mathrm{NN}}}=200 \mathrm{GeV}$. Left: NSD p+p and centrality-selected Au+Au collisions [5]. Right: central and minimum bias d+Au collisions, and same $\mathrm{p}+\mathrm{p}$ spectrum as left panel[6].

\section{SUPPRESSION OF INCLUSIVE CHARGED HADRONS}

Jets occasionally fragment with a single hadron carrying a large fraction of the total jet energy. The resulting inclusive hadron distributions exhibit power-law $\left(1 / p_{T}{ }^{n}\right)$ shapes characteristic of the underlying perturbative QCD (pQCD) processes. Figure 1 shows the event-normalized invariant inclusive charged hadron distributions as a function of $p_{T}$ for NSD $p+p$ and centrality-selected $\mathrm{Au}+\mathrm{Au}$ and $\mathrm{d}+\mathrm{Au}$ collisions [5, 6]. The centrality bins correspond to the indicated percentiles of the total cross section: $0-5 \%$ for $\mathrm{Au}+\mathrm{Au}$ indicates the most central and $60-80 \%$ the most peripheral collisions. Power-law shapes are clearly observed in all cases.

Nuclear effects on hadron production in $\mathrm{d}+\mathrm{Au}$ and $\mathrm{Au}+\mathrm{Au}$ collisions are measured through comparison to the $\mathrm{p}+\mathrm{p}$ reference spectrum using the ratio

$$
R_{A B}\left(p_{T}\right)=\frac{d^{2} N / d p_{T} d \eta}{T_{A B} d^{2} \sigma^{p p} / d p_{T} d \eta},
$$

where $d^{2} N / d p_{T} d \eta$ is the differential yield per event in the nuclear collision $A+B$, $T_{A B}=\left\langle\mathrm{N}_{\text {bin }}\right\rangle / \sigma_{\text {inel }}^{p p}$ describes the nuclear geometry, and $d^{2} \sigma^{p p} / d p_{T} d \eta$ for $\mathrm{p}+\mathrm{p}$ inelastic collisions is determined from the $\mathrm{p}+\mathrm{p}$ measurement. $\left\langle\mathrm{N}_{\mathrm{bin}}\right\rangle$ is the mean number of binary $\mathrm{NN}$ interactions for the given centrality class of $A+B$ collisions. In the absence of nuclear effects such as shadowing, the Cronin effect, or gluon saturation, hard process rates are expected to scale with $\left\langle\mathrm{N}_{\text {bin }}\right\rangle$, and $R_{A B}\left(p_{T}\right)=1$.

Figure 2, left panel, shows $R_{A B}\left(p_{T}\right)$ for centrality-selected $\mathrm{Au}+\mathrm{Au}$ relative to $\mathrm{p}+\mathrm{p}$ collisions. The error bars indicate the statistical and systematic uncertainties of the spectra, while the bands indicate the uncertainty due to the geometrical scaling factor $T_{A B}$. At the highest $p_{T}$, hadron suppression of approximately a factor 5 is observed for the most central collisions: inclusive hadron production is strongly suppressed at high 

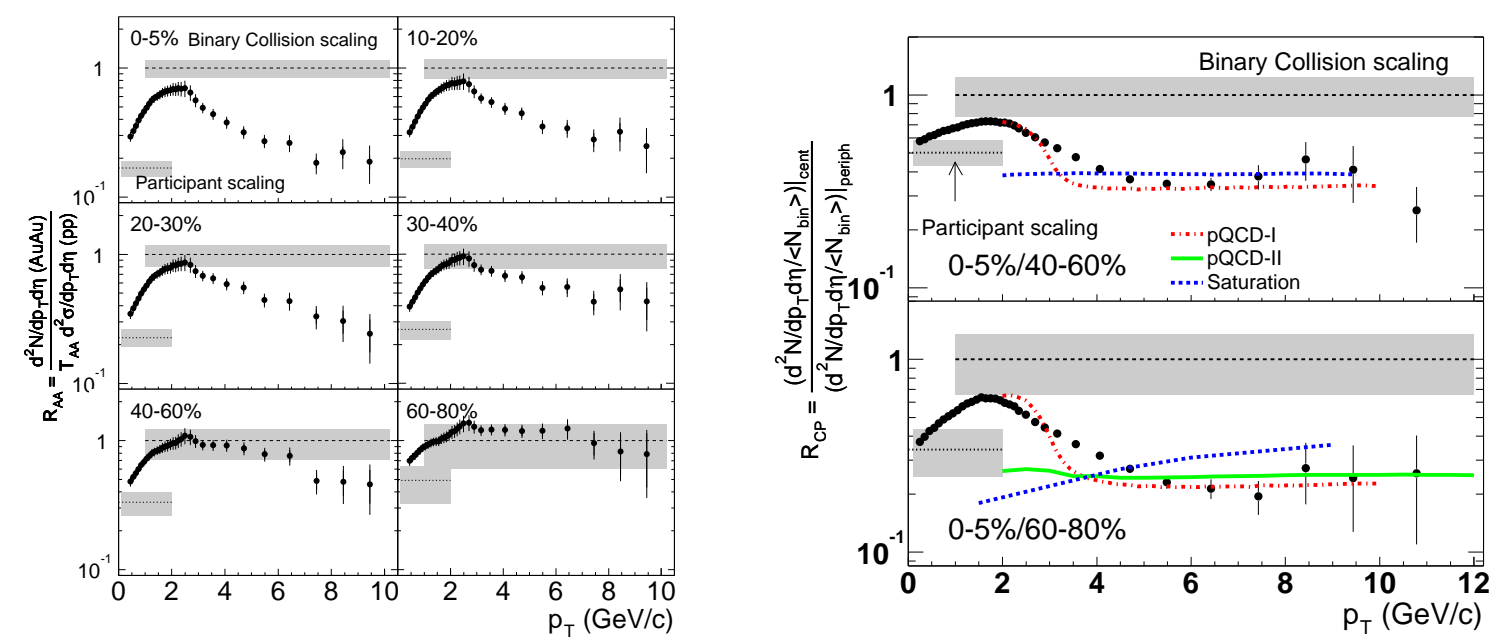

FIGURE 2. Left: $R_{A B}\left(p_{T}\right)$ (eq.11) for $\mathrm{Au}+\mathrm{Au}$ relative to $\mathrm{p}+\mathrm{p}$ collisions [5]. Right: $R_{C P}\left(p_{T}\right)$ from $\mathrm{Au}+\mathrm{Au}$ collisions[5].

$p_{T}$ in central $\mathrm{Au}+\mathrm{Au}$ collisions. For the most peripheral collisions $R_{A B}\left(p_{T}\right)$ is consistent with unity, while intermediate centralities interpolate smoothly between the extremes.

Figure 2] right panel, shows the related quantity $R_{C P}\left(p_{T}\right)$, the $\left\langle\mathrm{N}_{\text {bin }}\right\rangle$-scaled ratio of particle yields in central relative to peripheral collisions, which also exhibits large suppression of inclusive hadron production in central collisions. Figure 2 also shows the results of three theoretical calculations: two models based on pQCD incorporating shadowing, the Cronin effect and jet quenching in dense matter (pQCD-I[7], pQCDII[8]), and a model incorporating gluon saturation in the incoming Au nuclei[9]. The pQCD-based calculations contain one free parameter, the energy density for central collisions, which is fit to the data, yielding an initial density 30-50 times that of cold nuclear matter [7, 8]. These calculations then successfully describe the $p_{T}$ and centrality dependence of the inclusive suppression for $p_{T}>5 \mathrm{GeV} / \mathrm{c}$. The saturation model also describes the suppression and its $p_{T}$-dependence for the $0-5 \% / 40-60 \%$ ratio for $p_{T}>5$ $\mathrm{GeV} / \mathrm{c}$. These precision STAR data on inclusive charged hadron suppression, covering wide centrality and kinematic range, are well-described by widely differing models which attribute the suppression either to jet quenching in dense matter in the final state or to gluon saturation in the initial state.

In order to elucidate the particle-species dependence of the suppression, Fig. 3 shows $R_{C P}\left(p_{T}\right)$ for $K_{s}^{0}$ and $\Lambda+\bar{\Lambda}$ citeSTARStrangeRCP compared to the charged hadron $R_{C P}\left(p_{T}\right)$ already shown in Fig. 2. The mesons scale approximately as the charged hadrons throughout, while the baryons exhibit a pronounced enhancement in the region $2<p_{T}<4 \mathrm{GeV} / \mathrm{c}$. The origin of this behavior is at present not understood, though speculations include the Cronin effect and non-perturbative mechanisms such as baryon junctions. At $p_{T}=5 \mathrm{GeV} / \mathrm{c}$, all particle species are strongly suppressed by the same amount in central collisions. 


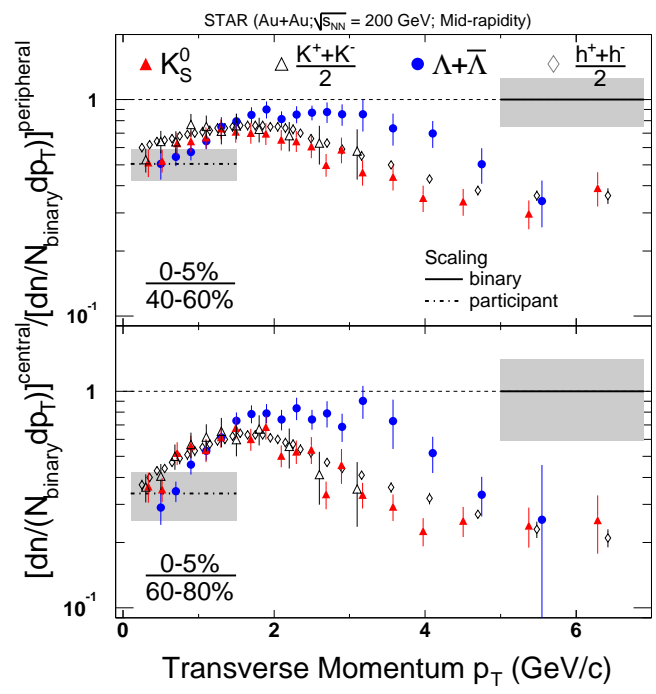

FIGURE 3. $R_{C P}\left(p_{T}\right)$ from $\mathrm{Au}+\mathrm{Au}$ collisions for $K_{s}^{0}$ and $\Lambda+\bar{\Lambda}[10]$, and for the charged hadron data also shown in Fig. 2] right panel.

\section{CORRELATIONS}

The angular correlations of pairs of high- $p_{T}$ charged particles can be used to study jets in the complex environment of heavy ion collisions[3]. Figure 4 shows the two-particle azimuthal distribution $D(\Delta \phi)$, defined as

$$
D(\Delta \phi) \equiv \frac{1}{N_{\text {trigger }}} \frac{1}{\varepsilon} \frac{d N}{d(\Delta \phi)},
$$

for peripheral (left panel) and central (right panel) $\mathrm{Au}+\mathrm{Au}$ collisions and for $\mathrm{p}+\mathrm{p}$ collisions (both panels). Only particles within $|\eta|<0.7$ are included in the analysis. $N_{\text {trigger }}$ is the number of particles within $4<p_{T}$ (trig) $<6 \mathrm{GeV} / \mathrm{c}$, referred to as trigger particles. The distribution results from the correlation of each trigger particle with all associated particles in the same event having $2<p_{T}<p_{T}$ (trig), where $\varepsilon$ is the tracking efficiency of the associated particles. The normalization uncertainties are less than $5 \%$.

In order to compare correlations in $\mathrm{Au}+\mathrm{Au}$ with $\mathrm{p}+\mathrm{p}$, the $\mathrm{p}+\mathrm{p}$ correlations are scaled up to the same pedestal value at $|\Delta \phi| \sim \pi / 2$ as $A u+A u$ and superposed with a $\cos (2 \Delta \phi)$ term that characterizes the azimuthal anisotropy in non-central $\mathrm{Au}+\mathrm{Au}$ collisions (elliptic flow [4]). The magnitude of this additional correlation is measured independently and is given by the second coefficient $v_{2}$ of a Fourier expansion of the azimuthal distribution relative to the orientation of the reaction plane of the event, for particles having $p_{T}<2 \mathrm{GeV} / \mathrm{c}$ [11]. Figure 4 shows that the correlation strength at small relative angle $(\Delta \phi \sim 0)$ in peripheral and central $\mathrm{Au}+\mathrm{Au}$ and at large relative angle $(\Delta \phi \sim \pi)$ in peripheral $\mathrm{Au}+\mathrm{Au}$ are very similar to the scaled correlations in $\mathrm{p}+\mathrm{p}$ collisions.

The near-side peaks $(\Delta \phi \sim 0)$ in all three collision systems are characteristic of jet fragmentation. The away-side peak $(|\Delta \phi| \sim \pi)$ from the back-to-back partner jet, apparent in $\mathrm{p}+\mathrm{p}$ and peripheral $\mathrm{Au}+\mathrm{Au}$ collisions is strongly suppressed in central $\mathrm{Au}+\mathrm{Au}$ 

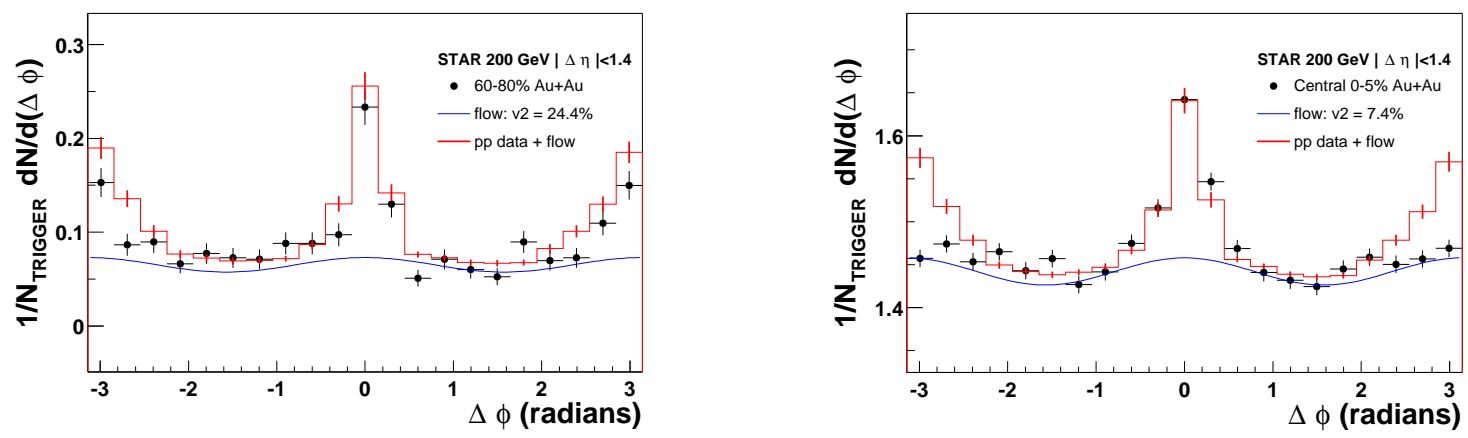

FIGURE 4. Azimuthal correlations for peripheral (left) and central (right) Au+Au collisions compared to the pedestal and flow-scaled correlations in $p+p$ collisions $[12]$.

collisions. These observations, together with the strong suppression of inclusive production and large elliptic flow at high $p_{T}$ [4], suggest a picture in which jets traversing the bulk of the medium produced in $\mathrm{Au}+\mathrm{Au}$ collisions are absorbed (strong jet quenching), and the observed jets are biased towards those generated on the surface and heading outwards. However, other explanations, in particular the gluon saturation picture, may also account for some or all of the observed phenomena. Experimentally, these very different scenarios can be discriminated through measurements of $\mathrm{d}+\mathrm{Au}$ collisions, where initial state effects in the Au nucleus remain but no final state dense medium is generated.

\section{D+AU COLLISIONS}

pQCD-based models predict an enhancement in the production of high- $p_{T}$ charged hadrons in $\mathrm{d}+\mathrm{Au}$ collisions relative to binary-scaled $\mathrm{p}+\mathrm{p}$ collisions and little change in the back-to-back correlation strength[13, 14], while one version of the saturation model[15] predicts an inclusive suppression in $\mathrm{d}+\mathrm{Au}$ of about $30 \%$ and possibly also suppression of the back-to-back strength due to a mono-jet contribution [16].

Figure 5 shows $R_{A B}\left(p_{T}\right)$ (left) and the pedestal subtracted two-particle azimuthal distributions (right) measured by STAR in minimum bias and central $\mathrm{d}+\mathrm{Au}$ collisions [6]. $R_{A B}\left(p_{T}\right)$ exceeds unity for $2<p_{T}<7 \mathrm{GeV} / \mathrm{c}$, consistent with expectations from the Cronin effect. However, no additional enhancement over $\mathrm{p}+\mathrm{p}$ is observed for central relative to minimum bias $\mathrm{d}+\mathrm{Au}$ collisions. In the top right panel, the azimuthal distributions are characterized by a fit to the sum of near-side and back-to-back Gaussian peaks plus a constant. The only significant difference between the $p+p$ and $d+A u$ correlations is the growth in the pedestal value [3]. The lower right panel shows the pedestaland flow-subtracted azimuthal distribution from central $\mathrm{Au}+\mathrm{Au}$ collisions along with the pedestal-subtracted distributions from central $d+A u$ and $p+p$. The near-side peak is similar in all three collision systems, while the away-side peak is suppressed only in central $\mathrm{Au}+\mathrm{Au}$ collisions. 

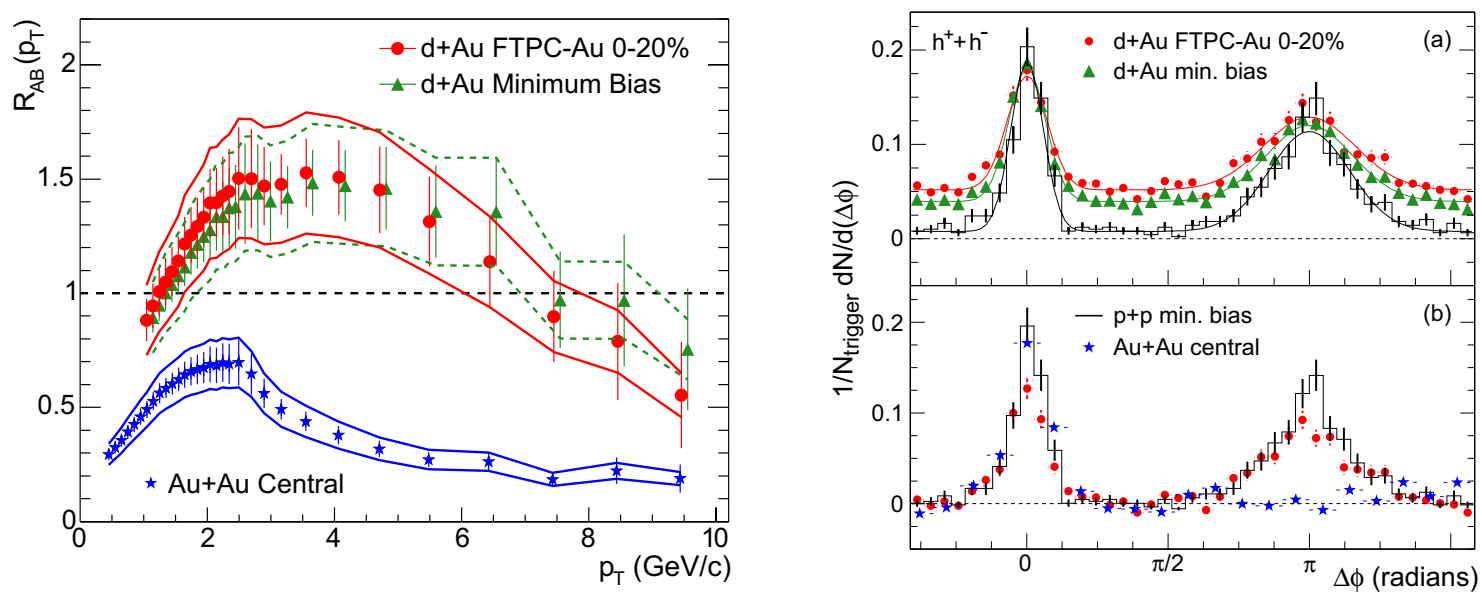

FIGURE 5. $R_{A B}\left(p_{T}\right)$ and two-particle azimuthal distributions in d+Au collisions[6]. The horizontal axes of the right panel are shifted by $\pi / 2$ relative to Fig. 4

\section{SUMMARY}

The strong suppression at high $p_{T}$ of the inclusive hadron yield and back-to-back correlations in central $\mathrm{Au}+\mathrm{Au}$ collisions at $\sqrt{s_{\mathrm{NN}}}=200 \mathrm{GeV}$ are not observed in d+Au collisions. The inclusive yield in $d+A u$ collisions is enhanced relative to binary-scaled $p+p$, consistent with expectations from the Cronin effect, and the back-to-back correlations show little variation relative to $\mathrm{p}+\mathrm{p}$. These results demonstrate conclusively that the striking suppression phenomena observed in central $\mathrm{Au}+\mathrm{Au}$ collisions are due to the interaction of high energy partons or their fragmentation products in the dense medium created in such collisions.

\section{REFERENCES}

1. R. Baier, D. Schiff and B. G. Zakharov, Ann. Rev. Nucl. Part. Sci. 50, 37 (2000); M. Gyulassy, I. Vitev, X.N. Wang, B. Zhang, nucl-th/0302077

2. K.H. Ackermann et al., Nucl. Instr. Meth. A499, 624 (2003).

3. M. Miller, these proceedings.

4. A. Tang, these proceedings.

5. J. Adams et al.(STAR Collaboration), nucl-ex/0305015

6. J. Adams et al.(STAR Collaboration), Phys. Rev. Lett. 91, 072304 (2003).

7. X.N. Wang, nucl-th/0305010 private communication. Calculations use model parameters $\mu_{0}=2.0$ $\mathrm{GeV}$ and $\varepsilon_{0}=2.04 \mathrm{GeV} / \mathrm{fm}$.

8. I. Vitev and M. Gyulassy, Phys. Rev. Lett. 89, 252301 (2002).

9. D. Kharzeev, E. Levin, and L. McLerran, Phys. Lett. 561, 93 (2003).

10. J. Adams et al.(STAR Collaboration), nucl-ex/0306007

11. C. Adler et al., Phys. Rev. Lett. 90, 032301 (2003).

12. C. Adler et al., Phys. Rev. Lett. 90, 082302 (2003).

13. I. Vitev, Phys. Lett. B562, 36 (2003).

14. X.N. Wang, Phys.Rev. C61, 064910 (2000); nucl-th/0303004

15. D. Kharzeev, E. Levin, and M. Nardi, hep-ph/0212316

16. D. Kharzeev, Nucl. Phys. A715, 35c (2003). 